\title{
Análisis de la Movilidad Espectral en Redes de Radio Cognitiva
}

\author{
Cesar Hernández ${ }^{(1,2) \star}$, Luis Pedraza ${ }^{(1,2)}$, Ingrid Páez ${ }^{(2)}$ y E. Rodriguez-Colina ${ }^{(3)}$ \\ (1) Universidad Distrital "Francisco José de Caldas", Facultad Tecnológica, Docente Asociado. Bogotá- \\ Colombia. (e-mail: cahernandezs@udistrital.edu.co, Ifpedrazam@udistrital.edu.co) \\ (2) Universidad Nacional de Colombia, Facultad de Ingeniería, Departamento de Sistemas e Industrial, \\ Doctorado en Ingeniería de Sistemas y Computación. Bogotá-Colombia. (e-mail: ippaezp@unal.edu.co) \\ (3) Universidad Autónoma Metropolitana Iztapalapa, Departamento de Ingeniería Eléctrica, Ciudad de \\ México-México. (e-mail: erod@xanum.uam.mx)
}

${ }^{*}$ Autor a quien debe ser dirigida la correspondencia

Recibido Jul. 20, 2015; Aceptado Sep. 25, 2015; Versión final Oct. 3, 2015, Publicado Dic. 2015

\section{Resumen}

Se presenta una revisión sobre la movilidad espectral en redes de radio cognitiva, describiendo el Handoff espectral, sus clasificaciones, sus enfoques actuales, sus criterios de decisión y sus métricas de evaluación de acuerdo con la literatura actual. La revisión se realizó a partir del análisis de publicaciones recientes de corriente principal con sus respectivas citas, tratando de proveer un marco referencial completo de la literatura actual sobre la movilidad espectral en redes de radio cognitiva. Los principales resultados determinan la importancia de soluciones escalables y la optimización de la detección y selección del canal objetivo, teniendo en cuenta la carga de tráfico, el comportamiento del usuario, los niveles de interferencia, la caracterización del espectro, el tipo de aplicación y la necesidad de múltiples canales de frecuencia. El estudio sugiere que se deben diseñar algoritmos adaptativos que permitan optimizar los recursos involucrados durante el Handoff espectral.

Palabras clave: acceso oportunista; asignación de espectro dinámica; Handoff espectral; radio cognitiva; redes inalámbricas móviles

\section{Analysis of Spectrum Mobility in Cognitive Radio Networks}

\begin{abstract}
A review of the spectrum mobility in cognitive radio networks, describing the spectrum Handoff, their classifications, current approaches, decision criteria and evaluation metrics according to the current literature is presented. The review was based on the analysis of recent mainstream publications with their respective citations, trying to provide a comprehensive reference framework of the current literature on the spectrum mobility in cognitive radio networks. The main results determine the importance of scalable and optimizing detection and targeting channel, taking into account the traffic load, the behavior of the user, the interference levels, and the characterization of the spectrum, the type of application and the need for multiple frequency channels. The study suggests that it is necessary to design adaptive algorithms to optimize the resources involved during the spectrum Handoff.
\end{abstract}

Keywords: opportunistic access; dynamic spectrum allocation; spectral Handoff; cognitive radio; mobile wireless networks 


\section{INTRODUCCIÓN}

Actualmente la cantidad de tráfico transportado por las redes inalámbricas está creciendo constantemente debido al aumento del número de usuarios y al incremento de las aplicaciones inalámbricas (López et al., 2015). Lo anterior, junto a una política de asignación fija de frecuencias ha producido una escasez en bandas de frecuencia disponibles. Sin embargo, los resultados de estudios realizados demuestran que ciertas bandas, como las de $50 \mathrm{MHz}$ a $700 \mathrm{MHz}$, están siendo subutilizadas, ya que sus ciclos útiles son prácticamente nulos y que en algunos casos los tiempos de utilización espectral son inferiores al $10 \%$ (Forero, 2012), en contraste con otras bandas como las asignadas a la red celular que actualmente se encuentran saturadas.

La Radio Cognitiva definida por la Unión Internacional de Telecomunicaciones (ITU), como "una radio o sistema que detecta y está al tanto de su entorno y se puede ajustar de forma dinámica y autónoma de acuerdo con sus parámetros de funcionamiento de radio", plantea como solución, el acceso dinámico al espectro, haciendo un uso oportunista e inteligente del espectro de frecuencia. Lo anterior, permite que un usuario secundario (SU) pueda utilizar un canal de una banda licenciada disponible, pero tan pronto llegue el usuario licenciado a ocupar dicho canal, el usuario secundario deberá dejarlo y buscar un nuevo canal disponible para continuar con su transmisión, a esto se le denomina movilidad espectral o Handoff espectral en la Radio Cognitiva (Akyildiz et al., 2006; Akyildiz et al., 2008). Durante el Handoff espectral es inevitable que la comunicación se rompa temporalmente, debido a que es necesario realizar un proceso de descubrimiento de nuevas bandas de frecuencias disponibles, por tanto, se requiere de un modelo de Handoff espectral que permita cambiar la actual transmisión del usuario secundario a una nueva banda espectral con la mínima degradación de calidad, reconfigurando sus parámetros de comunicación (Akyildiz et al., 2009; López et al., 2015).

De acuerdo con las investigaciones actuales (Akyildiz et al., 2006; Akyildiz et al., 2008; Akyildiz et al., 2009; Christian et al., 2012; Marinho y Monteiro, 2012; Rodriguez-Colina et al., 2011; Wang y Wang, 2009; Wang et al., 2012), el modelo de Handoff espectral es la característica clave para habilitar la transmisión continua de datos del usuario de radio cognitiva o usuario secundario, ya que a partir de este se podría reducir el número de cambios de canal durante la transmisión de un usuario secundario, así como su latencia, minimizando la degradación del canal (Christian et al., 2012). Actualmente existen varias propuestas de modelos de Handoff espectral, sin embargo, es importante analizar que la aplicación de un modelo de Handoff espectral depende fuertemente de las características de la red del usuario primario (Liu y Tewfik, 2014; Rahimian et al., 2014).

Las principales características de la radio cognitiva, que le confieren todas sus capacidades son la capacidad cognitiva y reconfigurabilidad. La capacidad cognitiva es la tecnología capaz de capturar la información de su entorno de radiofrecuencia para identificar los segmentos del espectro que no están siendo utilizados, seleccionar el mejor espectro posible y definir los parámetros de operación más adecuados con el objetivo de evitar la interferencia con otros usuarios (Akyildiz et al., 2006). La reconfigurabilidad es la capacidad de cambiar de forma dinámica, los diferentes parámetros de operación relacionados con la transmisión y/o recepción, como frecuencia, potencia y modulación, con el objetivo de habilitar la radio para ser programada dinámicamente para transmitir y recibir en una gran variedad de frecuencias en función del ambiente de radio, y usar diferentes tecnologías de acceso a la transmisión, soportado por su diseño de hardware (Akyildiz et al., 2006; Akyildiz et al., 2009).

Las redes de Radio Cognitiva (CRN) también imponen retos en la gestión del espectro, debido a la naturaleza fluctuante del espectro disponible y a los requerimientos de calidad de servicio de varias aplicaciones. Las cuatro principales funciones en la gestión del espectro son: detección del espectro, decisión de espectro, compartición de espectro y movilidad de espectro, la cuales conforman el ciclo cognitivo (Akyildiz et al., 2009; Akyildiz et al., 2008). El ciclo cognitivo, inicia con la detección del espectro, aquí los usuarios de radio cognitiva o usuarios secundarios, monitorizan el espectro para detectar las oportunidades espectrales (también denominados espacios blancos o huecos espectrales) a fin de no interferir con los usuarios licenciados o usuarios primarios (Akyildiz et al., 2009). En la decisión de espectro, después de que los huecos espectrales han sido identificados, los usuarios secundarios deben seleccionar el más adecuado de acuerdo con sus requerimientos de calidad de servicio.

Para tomar la anterior decisión se han desarrollado algoritmos que tienen en cuenta las características del canal de radio y el comportamiento estadístico de los usuarios primarios, entre otros factores (Akyildiz et al., 2009). Debido a que múltiples usuarios de radio cognitiva pueden intentar acceder al espectro, la función de compartición de espectro, proporciona la capacidad de compartir este recurso con múltiples usuarios secundarios, coordinando sus transmisiones para evitar colisiones e interferencias (Akyildiz et al., 2009). Finalmente la movilidad espectral se da cuando el usuario de radio cognitiva debe dejar el canal de 
frecuencia que está utilizando y continuar su comunicación en otro hueco espectral, debido a la llegada de un usuario primario o a la degradación de la calidad del canal (Akyildiz et al., 2009).

El resto del artículo está estructurado como sigue. En la sección II se realiza una descripción del Handoff espectral. En la sección III se describe las diferentes clasificaciones del Handoff espectral. En la sección IV se analizan los diferentes enfoques del Handoff espectral. En la sección V se presentan los criterios y métricas para el Handoff espectral. Finalmente en la sección VI se presentan las conclusiones.

\section{HANDOFF ESPECTRAL}

La movilidad de espectro o Handoff espectral se puede definir como el proceso en donde un usuario de radio cognitiva (usuario secundario) cambia su frecuencia de operación, cuando las condiciones de un canal se degradan o cuando un usuario primario (PU) aparece, ya que el primero se encuentra utilizando un canal licenciado (Akyildiz et al., 2006; Akyildiz et al., 2008). El concepto de Handoff de espectro en radio cognitiva difiere de los mecanismos tradicionales de las redes inalámbricas, en el sentido que hay dos tipos de usuarios con diferentes prioridades. Los usuarios primarios (con alta prioridad), los cuales tienen derecho a interrumpir la transmisión de los usuarios secundarios (con baja prioridad). En un Handoff tradicional los usuarios tienen la misma prioridad (Wang y Wang, 2008).

Durante la movilidad espectral es inevitable que la comunicación se rompa temporalmente, debido a que es necesario realizar un proceso de descubrimiento de nuevas bandas de frecuencias disponibles, por tanto, la movilidad espectral requiere de un modelo de Handoff de espectro que permita cambiar la actual transmisión del usuario secundario a una nueva banda espectral con la mínima degradación de calidad, reconfigurando sus parámetros de comunicación (Akyildiz et al., 2009). Cada vez que ocurre un Handoff de espectro los parámetros de operación de la red de radio cognitiva cambian para minimizar el impacto en la operación de los usuarios de radio cognitiva con el objetivo de satisfacer su calidad de servicio (QoS) (Wang et al., 2010).

\section{Causas y requerimientos de un Handoff espectral}

La necesidad de realizar un Handoff espectral en redes de radio cognitiva se puede presentar por alguna de las siguientes causas (Akyildiz et al., 2009):

El canal objetivo está siendo ocupado por un PU. En estrategias proactivas, donde el canal de backup se selecciona con anterioridad, y no se verifica su estado de ocupación al momento de realizar el cambio de canal, es posible que el SU lo encuentre ocupado por otro SU o por un PU.

Llegada de un PU al canal ocupado por el SU. Durante la transmisión de datos de un SU en un canal licenciado, un PU puede arribar y exigir la disponibilidad inmediata de dicho canal.

El canal ocupado por el SU se degrada. Aun sin la existencia de un PU, es posible que el SU deba realizar un cambio de canal debido a la degradación de la calidad del canal que está utilizando actualmente.

El SU interfiere al PU. Si durante el uso oportunista del canal licenciado por parte del SU, se interfiere al PU, es necesario realizar un Handoff espectral.

Variación del tráfico. Si la cantidad de tráfico en la banda de frecuencia aumenta significativamente es posible que el SU requiera realizar un cambio de canal buscando el balanceo de carga y garantizando mejores niveles de desempeño.

Movimiento del SU. Si el SU se desplaza geográficamente fuera de la zona de cobertura del nodo en un sistema centralizado, es necesario realizar un Handoff espectral.

La realización de un Handoff espectral puede afectar el desempeño y la calidad de servicio de las redes de radio cognitiva, por tanto, existen algunos requerimientos al momento de efectuar un Handoff espectral (Van et al., 2012):

Velocidad. El retardo de Handoff espectral debe ser lo suficientemente pequeño para no causar degradación o interrupción durante la comunicación.

Tasa de Handoff. Un elevado número de cambios de canal innecesarios afecta directamente el desempeño de la transmisión de datos, es necesario minimizar la tasa de Handoff. 
Confiabilidad. Minimizar el efecto del Handoff en la calidad de servicio, por ejemplo, en redes móviles la probabilidad de bloqueo de nuevas llamadas y la probabilidad de caída de llamadas actuales debe ser minimizada, así como balancear el tráfico entre celdas adyacentes.

Señalización. Es importante minimizar la señalización, ya que un alto volumen de señalización puede afectar significativamente el desempeño de la comunicación.

Éxito. Canales y recursos deben estar disponibles para garantizar un exitoso Handoff (Kaleem, 2012).

Múltiples Criterios de Handoff: La nueva red de acceso o canal debe ser seleccionada inteligentemente a partir de múltiples criterios, ya que seleccionar la mejor oportunidad espectral evita múltiples Handoffs (Kaleem, 2012).

\section{Fases y procedimiento del Handoff espectral}

El objetivo fundamental de cualquier modelo de Handoff espectral es la transición de una frecuencia a otra con la mínima degradación de calidad (Duan y Li, 2011). El Handoff espectral se desarrolla a partir de las tres fases siguientes (Ahmed et al., 2014): medición, decisión y ejecución.

Medición: en esta fase se realiza el descubrimiento de redes inalámbricas y la detección de oportunidades espectrales en dichas redes. Lo anterior se puede lograr a través de un enfoque centralizado o distribuido.

Decisión: en esta fase se toma la decisión del "cuando" y "donde" realizar el Handoff espectral, con base en los múltiples criterios y métricas que se hayan seleccionado.

Ejecución: en esta fase se realiza la transferencia de la actual conexión a la nueva, teniendo en cuenta los requerimientos del Handoff espectral mencionados anteriormente.

En el procedimiento del Handoff espectral, primero se asume que los usuarios secundarios SU1 y SU2, se comunican en el canal Ch1. Cuando el usuario primario aparece en el canal Ch1, SU1 lo detecta y se prepara para realizar el Handoff espectral. SU1 pausa su comunicación actual dentro de una duración predefinida. También se notifica a SU2 de la interrupción antes de otro intervalo de tiempo predefinido. Luego, SU1 y SU2, retoman la comunicación en el canal Ch2 seleccionado, o en el mismo canal Ch1. Finalmente, debido a que una trama puede ser interrumpida muchas veces durante el tiempo de transmisión, el Handoff espectral puede tener que ejecutarse muchas veces más.

Impacto de la movilidad espectral en RC:

La movilidad espectral tiene un impacto significativo en el desempeño de las redes de radio cognitiva. De acuerdo con la estrategia de Handoff espectral que se configure, el desempeño de las redes de radio cognitiva puede ser afectado por alguno de los siguientes factores: latencia, throughput, confiabilidad, señalización, interferencia PU, eficiencia energética, ancho de banda, SINR, calidad de servicio, y tasa de error:

Latencia: la magnitud de la latencia o retardo puede aumentar considerablemente con las estrategias de Handoff reactivas, debido al tiempo de detección de oportunidades espectrales empleado.

Throughput: el valor de la tasa de datos efectiva puede ser reducido debido a la capacidad de canal de la banda de frecuencia seleccionada por la estrategia de Handoff espectral.

Confiabilidad: una inadecuada toma de decisiones por parte de la estrategia de Handoff espectral puede contribuir a un mayor desbalanceo de la carga de tráfico de datos en la CRN. Esto puede afectar los parámetros de calidad de servicio como la probabilidad de bloqueo de nuevas llamadas y la probabilidad de caída de llamadas actuales.

Señalización: de acuerdo con la estrategia de Handoff espectral la cantidad de información de señalización puede aumentar considerablemente, sobre todo en las estrategias con canal de control común (CCC). La cantidad de información adicional reduce el nivel de la tasa de datos efectiva.

Interferencia PU: las estrategias de Handoff espectral reactivas siempre causan interferencia temporal al PU, la cual es proporcional al tiempo de detección de oportunidades espectrales empleado por el SU. La decisión de aumentar la potencia de transmisión para aumentar el Throughput, también aumenta la interferencia ocasionada a los PU o SU en canales de frecuencia adyacentes. 
Eficiencia energética: la ejecución de algoritmos muy complejos, el aumento innecesario de la potencia de transmisión, el tiempo de detección prolongado, entre otras, contribuyen a la reducción de la eficiencia energética del SU.

Ancho de banda: la utilización de múltiples canales de frecuencia para la transmisión de un solo SU, puede beneficiar el ancho de banda de este, pero también puede disminuir el ancho de banda potencial de otros SUs si no se cuenta con una métrica de justicia adecuada al nivel de tráfico de la red.

SINR: una inadecuada toma de decisiones por parte de la estrategia de Handoff espectral puede afectar la SINR tanto del SU como del PU. Lo anterior puede ser causado, por una pobre selección de canal, una aumento exagerado en la potencia de transmisión, el modo de transmisión empleado, un mal balanceo de carga.

Calidad de servicio: una pobre selección del canal de frecuencia por parte de la estrategia de Handoff espectral puede provocar que aplicaciones sensitivas al retardo tengan unos parámetros de QoS y QoE bajos.

Tasa de error: en las comunicaciones de datos, la tasa de error es una función de los siguientes parámetros: frecuencia de operación, modulación, potencia de transmisión y tecnología de comunicación, entre otros. La estrategia de Handoff espectral debe reconfigurar dichos parámetros al momento de realizar un cambio de canal.

\section{CLASIFICACION DEL HANDOFF ESPECTRAL}

Los modelos de Handoff espectral se pueden clasificar de tres formas diferentes, (1) de acuerdo con el momento en el que se selecciona el canal objetivo y se realiza el cambio de canal, (2) de acuerdo con el tipo de tecnología de la red a la que se hace el cambio de canal, y (3) de acuerdo con la forma de conexión a la nueva estación base en sistemas centralizados.

\section{Primera clasificación}

De acuerdo con el momento en el que se selecciona el canal objetivo y se realiza el cambio de canal, se ha determinado la existencia de cuatro tipos de modelos de Handoff espectral en las redes de radio cognitiva: el modelo no Handoff, el modelo puramente reactivo, el modelo puramente proactivo y el modelo hibrido (Tabla 1). Cada uno de las anteriores presenta ventajas y desventajas de acuerdo con los parámetros en los que se desarrolle la comunicación (Christian et al., 2012).

Modelo no Handoff espectral: EI SU se mantiene en el canal original y permanece inactivo hasta que el canal vuelve a quedar libre. Después de que el PU deja el canal licenciado, el SU retoma la transmisión de datos de nuevo. Este modelo causa una alta latencia al SU, ya que el retardo es tan largo como el PU este activo en el canal correspondiente. Este modelo puede ser adecuado para la transmisión de datos cortas que no sean sensibles al retardo (Marinho y Monteiro, 2012).

Modelo reactivo puro: En este caso el SU realiza la detección de espectro y el Handoff, después de la detección del usuario primario. La ventaja radica en que el SU puede obtener exactitud en el canal objetivo; sin embargo, esto produce una larga latencia debido a lo que demora la detección de espectro, así como una interferencia temporal al PU, ya que durante dicho tiempo coexistirán los dos en el mismo recurso espectral (Marinho y Monteiro, 2012).

Modelo proactivo puro: En este caso el SU realiza la detección de espectro y el Handoff, antes de la llegada del usuario primario (Marinho y Monteiro, 2012). Algunos de los beneficios de esta estrategia son: (1) decremento del tiempo y energía gastada para encontrar un canal disponible; (2) decremento del número de Handoff de espectro y perdida de la comunicación; (3) decremento de la interferencia causada a los usuarios primarios (Duan y Li, 2011). Sin embargo, el problema radica en que el Handoff de espectro proactivo está basado en procesos estocásticos, donde los usuarios de radio cognitiva utilizan las observaciones pasadas del canal para predecir la disponibilidad del espectro futuro, lo que podría llevar a tener que hacer muchas conmutaciones de canal innecesarias si el modelo de predicción es imperfecto (Akyildiz et al., 2006).

Modelo hibrido: Aquí se combina los modelos proactivo y reactivo, aplicando detección espectral proactiva y Handoff reactivo. 
Tabla 1: Comparación entre los modelos de Handoff espectral de la primera clasificación (Marinho y Monteiro, 2012)

\begin{tabular}{|c|l|l|l|l|}
\hline Estrategia & \multicolumn{1}{|c|}{ No Handoff } & \multicolumn{1}{|c|}{ Reactivo puro } & \multicolumn{1}{|c|}{ Proactivo puro } & \multicolumn{1}{c|}{ Hibrido } \\
\hline $\begin{array}{c}\text { Idea } \\
\text { Principal }\end{array}$ & Parar y esperar & $\begin{array}{l}\text { Monitoreo } \\
\text { reactivo y acción } \\
\text { reactiva }\end{array}$ & $\begin{array}{l}\text { Monitoreo proactivo y acción } \\
\text { proactiva }\end{array}$ & $\begin{array}{l}\text { Monitoreo proactivo y } \\
\text { acción reactiva }\end{array}$ \\
\hline Ventajas & $\begin{array}{l}\text { Muy baja interferencia } \\
\text { al PU }\end{array}$ & $\begin{array}{l}\text { Precisión en la } \\
\text { selección del } \\
\text { canal }\end{array}$ & $\begin{array}{l}\text { Muy rápida respuesta y } \\
\text { selección del canal inteligente }\end{array}$ & Respuesta rápida \\
\hline Desventajas & $\begin{array}{l}\text { Muy alta interferencia } \\
\text { al SU }\end{array}$ & Respuesta lenta & $\begin{array}{l}\text { Datos de la disponibilidad del } \\
\text { canal no actualizados y costo } \\
\text { computacional alto }\end{array}$ & $\begin{array}{l}\text { Datos de la } \\
\text { disponibilidad del canal } \\
\text { no actualizados }\end{array}$ \\
\hline Latencia & Alta (impredecible) & Media & Muy baja & Baja \\
\hline Dependencia & Actividad del PU & $\begin{array}{l}\text { Monitoreo del } \\
\text { espectro }\end{array}$ & $\begin{array}{l}\text { Canal de backup y precisión del } \\
\text { modelo de tráfico del PU }\end{array}$ & Canal de backup \\
\hline Contexto & $\begin{array}{l}\text { Red del PU con } \\
\text { transmisión de datos } \\
\text { cortos. }\end{array}$ & $\begin{array}{l}\text { Red del PU } \\
\text { general }\end{array}$ & Red del PU bien modelada & Red del PU general \\
\hline
\end{tabular}

\section{Segunda clasificación}

Con la gran diversidad de tecnologías y sistemas de comunicación inalámbrica, se hace indispensable garantizar la movilidad espectral entre redes heterogéneas. De acuerdo con el tipo de tecnología o arquitectura de la red a la cual se hace el cambio de canal, el Handoff espectral se puede clasificar de tres formas diferentes: Handoff horizontal, Handoff vertical, y Handoff diagonal (Ahmed et al., 2014; Al-Surmi et al., 2012; B`alan et al., 2012; Kassar et al., 2008; Stevens-Navarro y Wong, 2007).

Handoff horizontal: Ocurre cuando un SU se mueve fuera de la cobertura de una estación base a la de otra estación base pero dentro de la misma red de acceso (Van et al., 2012). En el ambiente de redes celulares también se le conoce como Handoff Intra-celda. El Handoff horizontal tradicional toma sus decisiones basadas en la evaluación de la medición de la fuerza de la señal recibida (RSS) para apoyar las comunicaciones "Always Best Connected". Este tipo de Handoff es ejecutado cuando el nivel de RSS está por debajo de un umbral específico. (Kaleem, 2012).

Handoff vertical: Ocurre cuando un SU se mueve se mueve fuera de la cobertura de una estación base a la de otra estación base de una red de acceso diferente, con diferente tecnología inalámbrica (Van Quang et al., 2012). En el ambiente de redes celulares también se le conoce como Handoff Inter-celda. En un ambiente inalámbrico heterogéneo, un SU debe moverse entre diferentes redes de acceso con funcionalidades y características distintas, por lo que el nivel de RSS no es suficiente para la toma de decisiones inteligentes al momento de realizar un Handoff espectral, es necesario incluir más criterios y métricas como el retardo, la tasa de datos efectiva, el ancho de banda, el patrón de tráfico, la potencia de transmisión, entre otras. Sin embargo, la inclusión de múltiples criterios incrementa la complejidad en la toma de decisiones, haciendo el proceso de Handoff vertical más desafiante. (Kaleem, 2012).

Handoff diagonal: Es la combinación del Handoff horizontal y el Handoff vertical (Ahmed et al., 2014). Ocurre cuando un SU se mueve se mueve fuera de la cobertura de una estación base a la de otra estación base de una red de acceso diferente, pero con igual tecnología inalámbrica.

\section{Tercera clasificación}

Otra clasificación más común en los sistemas de telefonía celular, es la que depende del número de estaciones base (BS) y/o puntos de acceso (AP) a los cuales el SU (o PU) está asociado en cualquier momento. De acuerdo a lo anterior, existen dos tipos de Handoff, Fuerte (Hard) y Suave (Soft) (Lin y AiChun, 2000).

Handoff Fuerte: Este tipo de Handoff, tambien conocido como "break before make", se da cuando al realizar el cambio de canal el SU (o PU) se desconecta de su actual estación base antes de conectarse a una nueva, y durante un tiempo no está conectado a ninguna estación base. De acuerdo a lo anterior, este tipo de Handoff solo utiliza un canal, lo que mejora la eficiencia espectral pero lo hace menos confiable que el 
Handoff Suave. Este tipo de Handoff se utiliza en GSM y en general en FDMA y TDMA cuando se usan diferentes rangos de frecuencias en canales adyacentes para minimizar las interferencias de canal.

Handoff Suave: Este tipo de Handoff, también conocido como "make before break", se da cuando al realizar el cambio de canal el SU (o PU) se conecta a una nueva estación base antes de desconectarse de la actual. De acuerdo a lo anterior, durante el Handoff Suave la comunicación se realiza en paralelo por los dos canales evitando la interrupción del enlace y proporcionando mayor confiabilidad a pesar del incremento de la complejidad en la implementación del mismo. Este tipo de Handoff se utiliza en CDMA y WCDMA.

\section{TIPOS DE ENFOQUE PARA HANDOFF}

Las redes de radio cognitiva pueden operar de acuerdo a varios enfoques básicos, cada uno de los cuales presenta ventajas y desventajas frente a su enfoque opuesto. En esta sección se estudiaran el enfoque con infraestructura frente al enfoque ad hoc, enfoque centralizado frente al distribuido, enfoque de asignación multicanal frente a asignación única, inclusión o no del PU, inclusión o no del SU, Canal de Control Común (CCC) dedicado o dinámico, segmentación o agrupamiento.

\section{Enfoque con infraestructura frente al enfoque ad hoc}

De acuerdo con la arquitectura de la red, las redes de radio cognitiva pueden clasificarse en redes basadas en infraestructura o redes de radio cognitiva ad hoc (CRAHNs) (Akyildiz et al., 2006). Las redes de radio cognitiva basadas en infraestructura tiene una entidad de red central similar a una estación base en redes celulares o un punto de acceso en las redes inalámbricas de área local. Por otro lado, las CRAHNs no tienen ninguna infraestructura, por tanto, un SU se comunica con otro SU a través de una conexión ad hoc, tanto en bandas espectrales licenciadas como no licenciadas. (Akyildiz et al., 2009).

En las redes con infraestructura, la información observada por cada SU alimenta la base de datos de la entidad central, de forma que esta pueda tomar decisiones inteligentes a fin maximizar los parámetros de comunicación, como el throughput, el ancho de banda, la relación señal a ruido y el balanceo de carga, entre otras, y eliminar o minimizar la interferencia hacia los PU. (Akyildiz et al., 2009). En el caso de las CRAHNs, los SU son responsables de tomar sus propias decisiones con base en observaciones locales únicamente, lo cual les impide realizar un uso eficiente de los recursos de la red entera. Para compensar el caso anterior, los SU pueden hacer uso de esquemas de colaboración, en los cuales cada SU intercambia su información de observación del espectro local, permitiéndoles tener un conocimiento aproximado de la red completa. (Akyildiz et al., 2009).

\section{Enfoque centralizado frente al enfoque distribuido}

Si las redes de radio cognitiva cuentan con infraestructura, pueden operar tanto con un enfoque centralizado como distribuido. En el enfoque centralizado existe una entidad central encargada de coordinar las funciones necesarias para la decisión y asignación del canal de frecuencia durante un Handoff espectral. Esta entidad central también conocida como estación base central (CBS), además cumple con la función de almacenar y procesar la información del ambiente de radio y del espectro, recibida por los SUs periódicamente o por demanda (Tragos et al., 2013).

Debido a que la CBS tiene un nivel mucho más elevado de procesamiento y autonomía energética que los SUs, desarrolla también funciones de monitorización del espectro de forma periódica. Esta información junto a la proporcionada por los SUs actualizan dinámicamente la base de datos central (CDB). La CBS procesa periódicamente la información de la CDB y calcula valores estimados de algunos parámetros de interés del espectro, como la probabilidad de ocupación espectral de los canales, la relación señal a ruido más interferencia (SINR) de los canales, el tiempo medio de disponibilidad de los canales, entre otros, los cuales permiten tomar decisiones más acertadas para la asignación de espectro. Lo anterior libera a los SUs de la carga computacional requerida para ejecutar un algoritmo robusto de asignación de espectro.

La principal ventaja en el enfoque centralizado es la observación y conocimiento global de la red, lo cual permite maximizar el throughput de la red, minimizar la interferencia entre los SUs, una asignación multicanal justa, y en general mejorar el nivel de desempeño de la red (Alnwaimi et al., 2011; Byun et al., 2008). Sin embargo, su mayor desventaja es la cantidad de información de señalización que se introduce a la red para coordinar los procedimientos de intercambio de información entre la CBS y los SUs. Además, si la CBS llegara a fallar se perdería el control sobre la asignación de espectro creando desequilibrio y un potencial caos en el sistema. (Tragos et al., 2013). 
En el enfoque distribuido no existe un CBS responsable de coordinar la asignación de espectro a los SUs. Por tanto los SUs toman decisiones por ellos mismos o de forma colaborativa con otros SUs vecinos, a través del intercambio de información y medidas dentro de un rango determinado (i.e. 2-3 saltos). Lo anterior hace que el enfoque distribuido sea más flexible y más eficiente que el enfoque centralizado, ya que puede adaptarse rápidamente a los posibles cambios o variaciones del ambiente de radio o de la red, porque solamente los SUs en el área afectada tendrán que hacer modificaciones e intercambiar información. Otra ventaja significativa del enfoque distribuido es la baja información de señalización requerida, ya que solo los nodos vecinos intercambian información. Entre las desventajas del enfoque distribuido se encuentra que las decisiones tomadas no son óptimas en razón a que los nodos solo tienen información de sus vecinos y no de la red completa, adicionando el hecho de que es posible que la información intercambiada no sea suficiente. (Tragos et al., 2013). También la falta de un soporte centralizado no permite obtener información completa de la topología de la red, provocando colisiones entre SUs e interferencia al PU (Giupponi y Pérez-Neira, 2008). En conclusión, en lo que respecta a la asignación de espectro, el enfoque distribuido puede tomar decisiones adecuadas en casos de baja carga de tráfico, pero para los casos contrarios, el enfoque centralizado toma mejores decisiones (Tragos et al., 2013).

Una solución hibrida entre el enfoque centralizado y el distribuido, es el enfoque de agrupamiento (cluster), el cual intenta eliminar las desventajas de cada uno. La red es dividida en $M$ clusters, cada uno con un enrutador principal (cluster head). Cada SU envía la información de espectro detectada a su correspondiente cluster head, los cuales se encargar de combinar la información completa y generar un vector de asignación de espectro final. Todos los cluster head intercambian su respectivo vector y de esta manera cada uno conoce el estado general de la red. Con la información de completa de la red cada cluster head decide que canal de frecuencia asignar y transmite esta información a todos los demás cluster head de la red. Este enfoque es más robusto contra fallas, hace un uso más eficiente del ancho de banda disponible, logra una mejor distribución de los usuarios en clusters y de la carga en múltiples canales, y reduce la sobrecarga de información de control, ya que los mensajes se intercambian a nivel del cluster y no de la red completa. (Alsarhan y Agarwal, 2009; Chen et al., 2007; Tragos et al., 2013).

\section{Enfoque multicanal frente al enfoque de canal único}

El enfoque de asignación de canal único, como se hace tradicionalmente, consiste en la asignación de una frecuencia central y un ancho de banda especifico alrededor de esa frecuencia. Lo anterior implica que los canales son contiguos en el espectro.

El caso de la asignación multicanal consiste en la agrupación de varios canales disponibles que no son adyacentes, para formar un solo canal. Este enfoque permite aumentar el ancho de banda del SU aprovechando las oportunidades espectrales con baja capacidad de canal. Una de las técnicas que permite el acceso simultáneo a varios canales de frecuencia es Discontiguous Orthogonal Frequency Division Multiplexing (DOFDM) (Chen et al., 2008). Lo anterior permite un uso más eficiente del espectro, ya que aprovecha los canales que por sí solos no son adecuados debido a su restringido ancho de banda, pero que en conjunto con otros canales similares puede satisfacer los requerimientos de un SU. La asignación multicanal puede incrementar significativamente la capacidad de la red y la tasa de datos de los SUs. (Tragos et al., 2013).

Sin embargo, la asignación multicanal también tiene limitaciones, debido a que los transreceptores no pueden agregar canales de frecuencia que se encuentran muy distanciados entre sí. En otras palabras, el span de agregación no es ilimitado, ya que generalmente cada transreceptor tiene un span máximo especificado, por ejemplo $12 \mathrm{MHz}$. Esto significa que si dos canales están separados más de $12 \mathrm{MHz}$, no pueden unirse en un solo canal. Por tanto, los algoritmos de asignación de espectro deben evitar crear pequeños canales de frecuencia que posteriormente no puedan agregarse. También es necesario analizar que la utilización de múltiples canales por parte de un solo SU, no deje sin oportunidades espectrales a otros SUs, por tanto, el algoritmo de asignación de espectro debería manejar alguna métrica de justicia para estos casos. (Tragos et al., 2013).

\section{Enfoque respecto a la inclusión o no de modelos de actividad del PU y SU}

De acuerdo con algunos trabajos (Akter et al., 2008; Rodriguez y Chahuan, 2015; Csurgai-Horvath y Bito, 2011; Rahimian et al., 2014) la precisión y exactitud en el modelado de la actividad de los PU y SU, es relevante para lograr un buen desempeño en las redes de radio cognitiva. Un buen modelo de la actividad del PU y SU, permite asignar inteligentemente cada canal de frecuencia disponible, optimizando el uso del espectro, maximizando los parámetros de comunicación, como la tasa de datos, el ancho de banda, la relación señal a ruido y el balanceo de carga, entre otros, y eliminando o minimizando la interferencia entre 
PU y SU. Sin embargo, la validez de dichos modelos generalmente está restringida a un determinado tiempo y lugar, para los cuales se diseñó.

Una estrategia de Handoff espectral reactiva, provoca interferencia temporal con el PU, debido a que durante el tiempo que tome realizar la movilidad del SU, coexistirán los dos en el mismo recurso espectral. Un buen modelo del PU puede evitar o minimizar esta interferencia, a través de una estrategia de Handoff espectral proactiva. Sin embargo, el problema radica en que dichos modelos están basados en procesos estocásticos, que utilizan las observaciones pasadas del canal para predecir la disponibilidad del espectro futuro, lo que podría llevar a tener que hacer muchas conmutaciones de canal innecesarias si el modelo de predicción es imperfecto. Pero no solo el modelo de actividad del PU es relevante, en (Akter et al., 2008) se propone un modelo de predicción para el SU, en razón a que en muchas oportunidades, múltiples SUs compiten por el mismo recurso de espectro, degradando la calidad de servicio. A través de la implementación de un filtro Kalman se logró realizar una significativa estimación del número de SUs en el futuro instantáneo.

\section{Enfoque respecto a un CCC dedicado o dinámico}

El CCC es uno de los requerimientos comunes en la asignación de espectro en CRN, el cual permite la coordinación entre SUs para la asignación del canal. EI CCC es un canal predefinido, para el intercambio de información de señalización, control y espectro entre los SUs, el cual puede ser global o local dependiendo del operador de red. A continuación se presentan las ventajas y desventajas de tener un CCC dedicado para la asignación del canal de frecuencia o dinámico.

En la literatura son más los trabajos de investigación que defienden la necesidad de la existencia de un CCC dedicado (Ding et al., 2010; Kim et al., 2010; Ma et al., 2007). El enfoque con CCC dedicado se puede dividir en dos situaciones: (1) cuando el CCC y el canal de datos del SU son el mismo, y (2) cuando el CCC se encuentra en un canal independiente del canal de datos del SU (Akyildiz et al., 2009). Cuando el canal de datos del SU y el CCC son el mismo, su utilización se comparte por periodos de tiempo fijos, y no es necesario un transreceptor adicional, ni se requiere cambiar la frecuencia para recibir o transmitir los mensajes. Sin embargo, cuando sea necesario realizar un Handoff espectral, el CCC desaparecerá. Aunque este enfoque proporciona una mayor eficiencia espectral al utilizar un solo canal de frecuencia, la cantidad de información del CCC reduce el throughput de la transmisión de datos del SU. (Akyildiz et al., 2009).

Cuando el canal de datos del SU y el CCC son independientes, el CCC no se ve afectado por la realización de un Handoff espectral, sin embargo, el costo de tener dos canales implica la necesidad de un transreceptor adicional y un incremento en el retardo. EI CCC independiente, puede ser global si es el mismo para todos los SUs en una CRN, o local es dedicado solamente a una pequeña área geográfica. En ambos casos, es necesario contar con un algoritmo de asignación de CCC que permita encontrar el canal óptimo del área geográfica de la CRN. (Akyildiz et al., 2009). Con respecto al enfoque del CCC dinámico existe muy pocos trabajos (Almasaeid y Kamal, 2010; Kondareddy et al., 2008). Aunque hace un uso más eficiente del espectro, es vulnerable al problema del nodo escondido, que puede llevar a un decremento del nivel de conectividad. Además, una selección dinámica de un CCC puede incrementar el nivel de retardo en la transmisión del SU. (Tragos et al., 2013).

\section{CRITERIOS DE HANDOFF}

En la asignación de espectro existen múltiples criterios que ayudan a tomar decisiones inteligentes. Sin embargo, cuantos y cuales criterios utilizar, depende del objetivo de cada estrategia de Handoff espectral. Para realizar un análisis objetivo, se analizaron todas las variables que intervienen durante el proceso de Handoff espectral y que pueden afectar el desempeño del mismo. Esto se logró a partir del análisis de cada una de las investigaciones consultadas alrededor del tema de movilidad espectral en redes de radio cognitiva (Ahmed et al., 2014; Masonta et al., 2013; Tragos et al., 2013). Las variables resultantes se clasificaron en tres categorías: criterios de información, criterios de control y criterios de evaluación. Las primeras proporcionan información que puede ayudar a una mejor toma de decisiones por parte del modelo de Handoff espectral; las segundas son aquellas variables que se pueden manipular por parte del modelo de Handoff espectral para obtener cambios significativos en las variables objetivo; las terceras son aquellas variables que determinan el nivel de desempeño del modelo de Handoff espectral.

\section{Criterios de información (supuestos)}

Estos criterios proporcionan la información necesaria para alimentar los algoritmos de toma de decisiones con base en los criterios de evaluación para la asignación de espectro a los SU en las CRN. Estos criterios varían de acuerdo con los objetivos de cada esquema de Handoff espectral. 
Calidad de Servicio: La calidad de servicio (QoS) es uno de los criterios más relevantes en los algoritmos de toma de decisión para la asignación de espectro de los SU en las CRN. La QoS permite diferenciar entre aplicaciones sensitivas al retardo y aplicación que no son sensitivas al retardo, en otras palabras permite clasificar las aplicaciones en tiempo real (RT) y mejor esfuerzo (BE). Con esta información el esquema de Handoff espectral puede tomar la decisión más adecuada en términos de las oportunidades espectrales disponibles.

Aplicaciones que no son sensibles al retardo pueden ser asignadas a oportunidades espectrales con nivel de disponibilidad intermedio a través de estrategias de No Handoff. Mientras que aplicaciones sensibles al retardo pueden ser asignadas a oportunidades espectrales con un nivel alto de disponibilidad a través de estrategias proactivas que minimicen el valor del retardo global, aquí el Throughput resulta más relevante que la BER. Lo anterior permite mejorar la eficiencia espectral.

Calidad del enlace: La calidad del enlace es otro criterio importante que normalmente se refleja a través de la tasa de bits errados (BER) y la relación señal a ruido (SNR), los cuales también afectan la QoS de la red. Esta última a veces se trabaja como relación señal a interferencia (SIR) o relación señal a ruido más interferencia (SINR).

La BER es el número de bits recibidos que han sido alterados debido al ruido y la interferencia, divididos por el número total de bits transmitidos durante un periodo de tiempo determinado (Ahmed et al., 2014). La tasa de error promedio del canal es un parámetro útil para estimar la caracterización del ambiente de radio en las CRN (Masonta et al., 2013). El nivel de BER está relacionado con el nivel de SNR, esto causa que la energía por bit transmitido sea una métrica importante en la estimación del error (Höyhtyä et al., 2008). A mayor SNR menor BER, sin embargo, es importante tener en cuenta que un mayor nivel de SNR generalmente implica un mayor nivel de potencia, lo cual causa mayor interferencia al PU. Por tanto, es necesario estimar un mínimo nivel de SNR que garantice cierto nivel de BER que no perjudique la comunicación (Tragos et al., 2013).

La calidad del enlace de comunicación en CRN puede variar dinámicamente en el tiempo y espacio. Por ello, es importante para el SU, monitorizar y analizar periódicamente la calidad del canal que está siendo utilizado, por ejemplo a través de la SNR. La SNR define la relación entre la potencia de la señal y la potencia del ruido. Es otro parámetro importante que afecta y refleja la QoS de la red. Usualmente RSS y SINR son consideradas similares pero, RSS está más inclinada a proveer conectividad y SINR a proveer QoS de la red (Ahmed et al., 2014).

Disponibilidad del canal: La disponibilidad de canal es un criterio que busca estimar la probabilidad de que un canal de frecuencia esté disponible. Algunos trabajos lo expresan como ocupación de canal, en cuyo caso se busca estimar la probabilidad de que un canal de frecuencia este ocupado. Este criterio es muy relevante en la asignación de espectro, ya que puede determinar las posibilidades de que una oportunidad espectral este libre para ser utilizada por un SU. Su valor es proporcional a la media aritmética de las medidas de disponibilidad obtenidas anteriormente por cada canal. Aquí resulta interesante determinar el periodo de tiempo a partir del cual se debe actualizar el valor promedio de la disponibilidad por canal. Un análisis de la serie de tiempo para esta variable podría estimar un intervalo de confianza para el periodo de tiempo de actualización. Lo anterior permite lograr una mejor eficiencia de energía, al no tener que correr el algoritmo de estimación del criterio de disponibilidad de canal continuamente.

Tiempo estimado de disponibilidad del canal: El tiempo estimado de disponibilidad es un criterio que busca determinar el valor promedio que un canal de frecuencia permanece disponible para un SU o PU. A diferencia del criterio de disponibilidad de canal, donde se determina la probabilidad de encontrar cierto canal libre, en el criterio de tiempo estimado de disponibilidad estima el tiempo medio durante el cual el canal permanece libre, una vez está disponible. Estos dos criterios no son sustitutos sino complementarios, ya que dos canales pueden tener la misma probabilidad de disponibilidad pero con distribuciones de tiempo de disponibilidad distintas. Seleccionar un canal con un tiempo estimado de disponibilidad alto, garantiza un menor número de Handoffs de espectro. Los autores en (Pla et al., 2010) proponen un modelo Markoviano para determinar la duración de las oportunidades espectrales. Una vez se ha modelado el tiempo ocioso del $\mathrm{PU}$, se aplican técnicas de matriz analítica para determinar la duración de las oportunidades espectrales para ser ocupadas por los SUs. La principal desventaja de esta técnica radica en su complejidad.

Patrón de tráfico del PU y SU: No hay ninguna garantía de que el canal de frecuencia seleccionado esté disponible durante toda la comunicación, por ello es beneficioso contar con un modelo que estime la actividad del PU en la CRN. En algunos trabajos se asume que se puede conocer el patrón de llegadas del PU, ya que no es aleatorio estadísticamente, debido a que depende del comportamiento humano. De esta 
forma se puede estimar la disponibilidad de canal y la longitud de tiempo que estará disponible (Akyildiz et al., 2009; Christian et al., 2012).

Los autores en (Wang et al., 2010) exponen varios trabajos que argumentan que el comportamiento de ocupación de los canales exhibe patrones que pueden ser modelados estadísticamente. Sin embargo, diferentes modelos pueden aplicar a diferentes aplicaciones como voz, video y paquetes de datos generales. Varios modelos asumen que la actividad del PU se puede modelar con inter-llegadas distribuidas exponencialmente (Chou et al., 2007; Kim y Shin, 2008; Lee y Akyildiz, 2008). En (Sriram y Whitt, 1986) el patrón de tráfico se modela como un proceso de dos estados ON-OFF, nacimiento y muerte, con sus respectivas tasas de nacimiento y muerte. Otros modelan la actividad del PU a partir del tiempo entre arribos de paquetes, la longitud de los paquetes y cantidad de paquetes.

Algunos trabajos se esfuerzan en modelar el comportamiento del PU en bandas específicas, a partir de datos experimentales (Pedraza et al., 2014). Los autores en (Willkomm et al., 2008) utilizan medidas reales de una red celular para modelar las características del uso del espectro por parte del PU. El análisis realizado muestra que el modelo de llegadas exponencialmente distribuidas es adecuado para capturar el tiempo de actividad del PU en llamadas no inalámbricas. Mientras que para las llamadas móviles dicho modelo no resulta útil, siendo más efectiva el uso de un modelo de caminata aleatoria aun en condiciones de tráfico alto. Los autores en (Hernández et al., 2009) validan el uso de series de tiempo como los modelos autoregresivo (AR), media móvil (MA), y autoregresivo integrado de media móvil (ARIMA), en el modelamiento y predicción de tráfico en redes Wi-Fi, obteniendo un alto nivel de precisión en el pronóstico de rango corto. En (Li y Zekavat, 2008) se presenta un trabajo sobre la predicción del patrón de tráfico para redes de radio cognitiva.

Los autores en (Wei et al., 2006) almacenan la información pasada y presente, de interés para una CRN, en un repositorio incluyendo la información de localización y tráfico del SU y PU. Es importante validar la información almacenada para asegurarse de que no es obsoleta. Esta colección de datos debe ser estadísticamente analizada y usada para modelar la actividad del PU en un canal de frecuencia dado (Issariyakul et al., 2009). De acuerdo con el aprendizaje maquinal, los SUs deberían ser hábiles para recordar las lecciones aprendidas en el pasado y actuar rápidamente en el futuro (Marinho y Monteiro, 2012). Idealmente el SU debería conocer el patrón de tráfico del PU y seleccionar la estrategia de Handoff más adecuada. Cuando el patrón de tráfico del PU cambie, SU debería observar el cambio y adaptar una estrategia de Handoff adecuada. (Akyildiz et al., 2009; Christian et al., 2012). Las estrategias de Handoff espectral futuras deberían considerar un factor de aprendizaje.

Fecha y hora: La utilización del espectro depende del tiempo y del espacio. El criterio de fecha y hora puede ser un buen dato de información para estimar el nivel de tráfico y congestión de la red, a partir de las estadísticas de la historia de información pasada (Hernández et al., 2009; Hernandez et al., 2013; Issariyakul et al., 2009; Wei et al., 2006). Una base de datos de estadísticas pasadas y recientes, podrían permitir estimar la disponibilidad y acceso al espectro con cierto nivel de precisión. Las series de tiempo son una herramienta muy útil en este contexto (Hernández et al., 2009).

Geo-Localización: De acuerdo con la naturaleza de las redes inalámbricas, la disponibilidad de espectro no solamente cambia con el tiempo sino también con la movilidad espacial (Duan y Li, 2011). Conocer la posición exacta de los SUs y PUs es una ventaja en la toma de decisiones para la asignación de espectro. Esta información puede mejorar las estrategias para evitar la interferencia al PU y reducir la tasa de Handoff espectral, al determinar una distancia umbral a partir de la cual se deba realizar el cambio de canal. En zonas rurales, debido a la baja demanda de espectro, es posible utilizar un ancho de banda más amplio. Este conocimiento puede ser útil para futuras predicciones de oportunidades espectrales y la caracterización del ambiente de radiofrecuencia.

Capacidad del canal o ancho de banda disponible: Muchos trabajos se enfocan en parámetros como la tasa de datos, el retardo, el nivel de interferencia, la tasa de error o la tasa de cambios de canal, los cuales son relevantes para la eficiencia espectral. Sin embargo, la capacidad de canal es otra variable de interés en la asignación de espectro, ya que algunas aplicaciones requieren un mínimo ancho de banda para mantener sus parámetros de calidad de servicio. En los sistemas de multiplexación por división de frecuencia ortogonal (OFDM), cada banda espectral tiene un diferente ancho de banda que consiste de varias subportadoras (Masonta et al., 2013).

RSS: También conocida como indicador de fuerza de la señal recibida (RSSI) y fuerza relativa de la señal recibida (RRSS), es un factor tradicional e importante para tomar decisiones de Handoff espectral. RSS provee información acerca del nivel de potencia que está siendo recibido por la antena, el cual decrementa 
cuando el usuario se aleja del actual punto de acceso a la red. (Ahmed et al., 2014). Este criterio permite determinar el momento en el que se hace necesario realizar un cambio de canal.

Costo monetario: Las redes que funcionan sobre bandas de espectro licenciadas, proveen ciertos servicios a los usuarios a cambio de un costo monetario. El valor del costo monetario dependerá principalmente del recurso de ancho de banda y del tiempo durante el cual se utilizó. Si dos redes proveen la misma calidad de servicio entonces la red con el costo más bajo será la preferida por los SUs (Ahmed et al., 2014). Debido a lo anterior, el valor del costo monetario es una variable de información de interés para el algoritmo de asignación de espectro.

Preferencias de usuario: Las preferencias de los usuarios pueden ser definidas con base en la red preferida dentro de las disponibles, para la ejecución de las aplicaciones. Las preferencias de los usuarios también se pueden definir a partir de las prioridades asignadas a cada aplicación, las cuales pueden ser alta o baja. Usualmente los usuarios prefieren conexiones con alto ancho de banda, bajo costo y amplia cobertura, entre otras. (Ahmed et al., 2014). En la mayoría de los trabajos que analizan las preferencias de los usuarios, utilizan funciones de utilidad que permiten describirlas y manipularlas matemáticamente para encontrar óptimos.

Seguridad de la red: La seguridad es uno de los temas más relevantes en la convergencia de redes, debido a que cada red tiene sus propias opciones de seguridad. El proceso de Handoff espectral requiere proveer seguridad y privacidad contra intercepciones ilegales o ataques de denegación de servicio. (Ahmed et al., 2014).

\section{Criterios de control}

Estos criterios permiten en cierta medida, adaptar el nivel desempeño de la transmisión de datos, a las variaciones o cambios de las condiciones iniciales del ambiente o la comunicación. La adaptación se materializa a través de la reconfiguración de los parámetros de comunicación como la frecuencia de operación, la potencia de transmisión, la modulación, el esquema de codificación y el ancho de banda, entre otros.

Frecuencia de operación: La frecuencia de operación es uno de los parámetros reconfigurables claves en las redes de radio cognitiva. Este permite modificar dinámicamente la frecuencia central de la transmisión de datos, debido al cambio en el ambiente de radiofrecuencia. En (Weingart et al., 2007) se propone un modelo predictivo para seleccionar dinámicamente la configuración adecuada. Dicho modelo de predicción debe ser actualizado continuamente para garantizar una predicción confiable en tiempo real basado en un objetivo determinado, a partir de un conjunto de posibles configuraciones. (Masonta et al., 2013).

Existen compromisos entre la frecuencia de operación y algunas medidas de transmisión; si la frecuencia aumenta entonces también aumentan las pérdidas por trayectoria, y si se aumenta la potencia de transmisión para compensar dichas pérdidas, entonces también aumenta la interferencia al PU (Akyildiz et al., 2008).

Potencia de transmisión: El control sobre la potencia de transmisión permite reducir la interferencia co-canal, maximizar la capacidad de la red y minimizar el uso de energía (Han y Liu, 2008). Sin embargo, lograr un efectivo control de potencia de transmisión es una tarea bastante compleja, debido a los compromisos entre este parámetro y el nivel de interferencia generado al PU. Si se aumenta la potencia de transmisión, mejora la calidad de servicio del SU, pero al mismo tiempo aumenta el nivel de interferencia al PU. Si se disminuye la potencia de transmisión, disminuye la interferencia al PU, pero también disminuye la SINR recibida por el SU. La potencia de transmisión se debe seleccionar cuidadosamente para mantenerla por encima de la sensibilidad de los SU y esperar hasta que aparezca un canal disponible que permita aumentarla. (Masonta et al., 2013; Tragos et al., 2013)

Modulación y esquema de codificación: EI SU debería reconfigurar su esquema de modulación y codificación para adaptarse a los cambios en las condiciones de canal o en los requerimientos del usuario (Akyildiz et al., 2006). Un cambio en el nivel de interferencia puede modificar la SINR del SU, produciendo un aumento de la BER. Sin embargo, el nivel requerido en la BER se puede mantener ajustando el esquema de modulación.

Los autores en (Hong et al., 2008) proponen un esquema de transmisión adaptativa basado en un escenario de transmisión simplificado para redes de radio cognitiva. Dicho esquema selecciona adaptativamente la modulación que provee el máximo Throughput para el SU de acuerdo con los canales disponibles. 
Ancho de banda (Número de canales a utilizar): La definición de canal se puede interpretar como una frecuencia central asociada a un determinado ancho de banda. El ancho de banda se refiere a la amplitud del espectro sobre el cual un transreceptor propaga su señal. Dependiendo de las características del ambiente de radio y de la aplicación utilizada, un SU puede comunicarse sobre anchos de banda angostos 0 amplios (Masonta et al., 2013). Para la operación sobre redes heterogéneas es importante que los SU puedan soportar y adaptarse a anchos de banda distintos. La adaptación al ancho de banda puede ser muy útil en zonas rurales, donde se pueden tolerar tasas de datos bajas a cambio de un mayor alcance o una reducción de la potencia necesaria. (Masonta et al., 2013). En el caso de selección de múltiples canales, hay que tener en cuenta que los transreceptores no pueden agregar fragmentos de espectro que estén muy lejos entre sí, ósea que el span de agregación de bandas no es infinito (Tragos et al., 2013).

Tecnología de comunicación: La capacidad de reconfigurabilidad de las redes de radio cognitiva, les debería permitir la interoperabilidad en una gran amplia diversidad de sistemas de tecnologías de comunicación diferentes, tales como GSM, LTE, WiMAX, Wi-Fi, entre otras. Actualmente existen varias propuestas de estrategias de Handoff de espectro vertical (Ramirez-Perez y Ramos-R, 2013; Stevens-Navarro et al., 2012) para realizar el cambio de canal de frecuencia entre tecnologías de comunicación distintas.

\section{Criterios de evaluación y objetivo}

Calidad de Servicio: La calidad de servicio comprende varias métricas de evaluación para medir el desempeño del Handoff espectral en las redes de radio cognitiva, tales como el ancho de banda de transmisión, el retardo y el throughput, entre otras. En cuento al ancho de banda, la estrategia de Handoff espectral que se ejecute, podría estar en la capacidad de realizar una transmisión multicanal, aumentando considerablemente el ancho de banda de transmisión y mejorando significativamente el desempeño de la aplicación ejecutada.

El retardo end to end es el tiempo total de la entrega de un paquete medido desde la fuente hasta el destino, y tiene en cuenta los diferentes tipos de retardo que se pueden presentar, tales como: detección, ejecución, cambio, entre otros. En el caso del retardo de cambio, es el tiempo que necesita un SU para moverse de una frecuencia a otra, este retardo puede ser del orden de $10 \mathrm{~ms}$ para un cambio de $10 \mathrm{MHz}$ en frecuencias de hasta $3 \mathrm{GHz}$, y es proporcional a la diferencia entre la frecuencia central actual y la nueva. (Tragos et al., 2013). En el caso de los algoritmos de aprendizaje, la velocidad de convergencia es deseable por dos razones: mejor adaptación al ambiente dinámico y menor costo y overhead (Xu et al., 2013). Muchos trabajos se enfocan en mejorar o maximizar el Throughput, ignorando otras medidas de QoS como la distorsión en aplicaciones multimedia (Marinho y Monteiro, 2012). En (Ferro et al., 2011) se realiza un trabajo sobre la Maximización de Throughput, sin embargo, considerar solo el Throughput no es suficiente en aplicaciones sensibles al retardo como VolP (Tragos et al., 2013).

Suposiciones: La mayoría de modelos asumen que los valores de los criterios son precisos. Lo cual no es tan válido, ya que parámetros como el ancho de banda disponible, el jitter, retardo, entre otros, no pueden ser estimados o tienen un porcentaje de error. (Ahmed et al., 2014).

Tasa de Handoff de Espectro: El número de Handoffs de espectro afecta directamente el desempeño de las transmisiones del SU y puede reducir significativamente el throughput promedio del SU. Los Handoffs de espectro también se podrían clasificar como exitosos, fallidos e innecesarios. El Handoff fallido se da cuando una red A inicia el Handoff, pero la red objetivo B aún no está lista para la solicitud de Handoff debido a la deficiencia de recursos. La falla se debe a que no hay disponibilidad de canales en la red $B$ (Ahmed et al., 2014). Los denominados Handoffs innecesarios o Efectro Ping-Pong, son handoffs recurrentes que podrían causar un consumo extra de los recursos de la red, y que pueden afectar otras funcionalidades de la red (Ahmed et al., 2014).

Nivel de interferencia al PU / SU: Controlar la interferencia es esencial para lograr un máximo desempeño en redes inalámbricas, ya que la interferencia afecta directamente las capacidades de recepción de los clientes y la BER. La asignación de canal es uno de los mecanismos básicos que controla la interferencia en redes inalámbricas, por ejemplo la interferencia entre canales adyacentes (Tragos et al., 2013). Debido a lo anterior, no es recomendable utilizar canales de frecuencia adyacentes por cuestiones de interferencia, pero tampoco muy lejanos. La interferencia afecta tanto al transmisor como al receptor. La interferencia disminuye la SINR y esto a su vez causa una reducción de la tasa de transmisión, reduce la utilización de recursos, la perdida de paquetes, retardo de paquetes, entre otras. (Ahmed et al., 2014). Las soluciones para el Handoff espectral deberían ser escalables y depender no solo de la carga de tráfico y el comportamiento del usuario, sino también de los niveles de interferencia (NSF Workshop, 2009). 
Fairness: La transmisión multicanal en redes inalámbricas es utilizada para mejorar la capacidad de canal, maximizando el Throughput (Akyildiz et al., 2009). El criterio de maximizar el Throughput del SU o de la red puede causar un unfairness en la distribución del espectro, por ejemplo en casos donde un SU puede seleccionar múltiples canales y otros ninguno. Para el caso anterior es mejor un enfoque centralizado ya que ayuda a lograr el fairness mucho mejor. Para ser más justos se podría considerar grupos separados de SUs de acuerdo con sus requerimientos de QoS o usar prioridades para los usuarios con diferente QoS. Otra opción es enfocarse en maximizar el mínimo AB promedio por flujo. (Tragos et al., 2013). De acuerdo con lo anterior, existe un trade off entre el Throughput y el fairness.

Eficiencia espectral: La eficiencia espectral está dada por la razón entre el ancho de banda del espectro utilizado y la tasa de datos de transmisión efectiva (MHz/Mbps), en otras palabras, cuantos bits caben en un Hertz. La detección de espectro disminuye la eficiencia espectral, por tanto, es necesario minimizar el tiempo de detección. La velocidad de detección y la exactitud de detección son dos factores importantes en la detección de espectro para una movilidad espectral eficiente. De hecho, existe un trade off entre las dos.

Eficiencia energética: En CRAHN la eficiencia de energía llega a ser la mayor restricción debido a los limitados recursos de los nodos de CR. La frecuente actualización de la información espectral y la detección de espectro, utilizan una potencia significativa (Akyildiz et al., 2009). Dispositivos con restricciones de energía necesitan estrategias reactivas (Akyildiz et al., 2009). Actualmente todos los trabajos transforman el problema de maximización de la eficiencia de energía en un problema de minimización de la potencia de transmisión del SU (Tragos et al., 2013).

\section{CONCLUSIONES}

De acuerdo con el análisis desarrollado se hace evidente la necesidad de diseñar algoritmos adaptativos que permitan optimizar los recursos involucrados durante el Handoff espectral. Así mismo la colaboración distribuida juega un papel clave para el Handoff espectral en las redes ad hoc.

Las soluciones para el Handoff espectral deberían ser escalables y optimizar la detección y selección del canal objetivo, teniendo en cuenta la carga de tráfico, el comportamiento del usuario, los niveles de interferencia, la caracterización del espectro, el tipo de aplicación y la necesidad de múltiples canales de frecuencia.

En la práctica las redes de radio cognitiva deben enfrentar retos de tipo heterogéneo, como por ejemplo las infraestructuras de las redes actuales, por tal razón, se evidencia la necesidad de contar con un modelo de Handoff espectral capaz de adaptar su principio de funcionamiento para lograr el mejor desempeño en la movilidad espectral de los usuarios secundarios.

\section{AGRADECIMIENTOS}

Este trabajo fue realizado con la financiación del Centro de Investigaciones y Desarrollo Científico de la Universidad Distrital Francisco José de Caldas. Los autores también agradecen la colaboración de la Universidad Nacional de Colombia y la Universidad Autónoma Metropolitana de México.

\section{REFERENCIAS}

Ahmed, A., Boulahia, L. M. y Gaïti, D; Enabling vertical handover decisions in heterogeneous wireless networks: A state-of-the-art and a classification. IEEE Communications Surveys and Tutorials, 16(2), 776$811(2014)$

Akter, L., Natarajan, B. y Scoglio, C.; Modeling and Forecasting Secondary User Activity in Cognitive Radio Networks. In 17th International Conference on Computer Communications and Networks. (2008)

Akyildiz, I. F., Lee, W. Y., Vuran, M. C. y Mohanty, S.; NeXt generation/dynamic spectrum access/cognitive radio wireless networks: A survey. Computer Networks, 50(13), 2127-2159 (2006)

Akyildiz, I. F., Lee, W. Y. y Chowdhury, K. R.; CRAHNs: Cognitive radio ad hoc networks. Ad Hoc Networks, $7(5), 810-836(2009)$

Akyildiz, I. F., Lee, W. Y., Vuran, M. C. y Mohanty, S.; A survey on spectrum management in cognitive radio networks. IEEE Communications Magazine, 46(4), 40-48 (2008)

Almasaeid, H. M. y Kamal, A. E.; Receiver-Based Channel Allocation for Wireless Cognitive Radio Mesh Networks. In IEEE Symposium on New Frontiers in Dynamic Spectrum, 1-10 (2010) 
Alnwaimi, G., Arshad, K. y Moessner, K.; Dynamic spectrum allocation algorithm with interference management in co-existing networks. IEEE Communications Letters, 15(9), 932-934 (2011)

Alsarhan, A. y Agarwal, A.; Cluster-Based Spectrum Management Using Cognitive Radios in Wireless Mesh Network. In 18th Internatonal Conference on Computer Communications and Networks. 1-6 (2009)

Al-Surmi, I., Othman, M. y Ali, B. M.; Mobility management for ip-based next generation mobile networks: Review, challenge and perspective. J. Netw. Comput. Appl., 35(1), 295-315 (2012)

Arturo B. Rodriguez, L. J. R. y Chahuan, J.; Nueva Generación de Heurísticas para Redes de Fibra Óptica WDM (Wavelength División Multiplexing) bajo Tráfico Dinámico, doi: 10.4067/S0718-07642015000500017, Inf. tecnol. (en línea), 26(5), 135-142 (2015)

B`alan, I. M., Moerman, I., Sas, B. y Demeester, P.; Signalling minimizing handover parameter optimization algorithm for Ite networks. Wirel. Netw, 18(3), 295-306 (2012)

Byun, S. S., Balasingham, I. y Liang, X.; Dynamic spectrum allocation in wireless cognitive sensor networks: Improving fairness and energy efficiency. In IEEE Vehicular Technology Conference. (2008)

Chen, D., Zhang, Q. y Jia, W.; Aggregation aware spectrum assignment in cognitive ad-hoc networks. In Proceedings of the 3rd International Conference on Cognitive Radio Oriented Wireless Networks and Communications, CrownCom. (2008)

Chen, T., Zhang, H., Maggio, G. M. y Chlamtac, I.; CogMesh: A cluster-based cognitive radio network. In 2nd IEEE International Symposium on New Frontiers in Dynamic Spectrum Access Networks.168-178 (2007)

Chou, C. T., Shankar N, S., Kim, H. y Shin, K. G.; What and how much to gain by spectrum agility? IEEE Journal on Selected Areas in Communications, 25(3), 576-587 (2007)

Christian, I., Moh, S., Chung, I. y Lee, J.; Spectrum mobility in cognitive radio networks. IEEE Communications Magazine, 50(6), 114-121 (2012)

Csurgai-Horvath, L. y Bito, J.; Primary and secondary user activity models for cognitive wireless network. In 11th International Conference on Telecommunications. 301-306 (2011)

Ding, L., Melodia, T., Batalama, S. N., Matyjas, J. D. y Medley, M. J.; Cross-layer routing and dynamic spectrum allocation in cognitive radio ad hoc networks. IEEE Transactions on Vehicular Technology, 59(4), 1969-1979 (2010)

Duan, J. y Li, Y.; An optimal spectrum handoff scheme for cognitive radio mobile Ad Hoc networks. Advances in Electrical and Computer Engineering, 11(3), 11-16 (2011)

Ferro, R., Pedraza, L. y Hernandez, C.; Maximización del Throughput en una red de radio cognitiva basado en la probabilidad de falsa alarma. Tecnura, 15(30), 64-70 (2011)

Forero, F.; Detección de códigos de usuarios primarios para redes de radio cognitiva en un canal de acceso DCMA. Colombia. (2012)

Giupponi, L. y Pérez-Neira, A. I.; Fuzzy-based spectrum handoff in cognitive radio networks. In 3rd International Conference on Cognitive Radio Oriented Wireless Networks and Communications, CrownCom. (2008)

Han, Z. y Liu, K. J. R.; Resource allocation for wireless networks: basics, techniques, and applications (1st edition). United Kingdom: Cambridge university press. (2008)

Hernández, C., Salcedo, O. y Pedraza, L.; An ARIMA model for forecasting Wi-Fi data network traffic values. Ingeniería E Investigación, 29(2), 65-69 (2009)

Hernandez, C., Salgado, C. y Salcedo, O.; Performance of multivariable traffic model that allows estimating Throughput mean values. Revista Facultad de Ingenieria Universidad de Antioquia, (67), 52-62 (2013)

Hong, M., Kim, J., Kim, H. y Shin, Y. An Adaptive Transmission Scheme for Cognitive Radio Systems Based on Interference Temperature Model. In 5th IEEE Consumer Communications and Networking Conference. 69-73 (2008) 
Höyhtyä, M., Mustonen, M., Sarvanko, H., Hekkala, A., Katz, M., Mämmelä, A. y Kautio, A.; Cognitive radio : An intelligent wireless communication system. Research Report VTT-R-02219-08. (2008)

Issariyakul, T., Pillutla, L. S. y Krishnamurthy, V.; Tuning radio resource in an overlay cognitive radio network for TCP: Greed isn't good. IEEE Communications Magazine, 47(7), 57-63 (2009)

Kaleem, F.; VHITS : Vertical Handoff Initiation and Target Selection in a Heterogeneous Wireless Network. (2012)

Kassar, M., Kervella, B. y Pujolle, G.; An overview of vertical handover decision strategies in heterogeneous wireless networks. Computer Communications, 31(10), 2607-2620 (2008)

Kim, H. y Shin, K. G.; Efficient discovery of spectrum opportunities with MAC-layer sensing in cognitive radio networks. IEEE Transactions on Mobile Computing, 7(5), 533-545 (2008)

Kim, W., Kassler, A. J., Di Felice, M. y Gerla, M.; Urban-X: Towards distributed channel assignment in Cognitive Multi-Radio Mesh Networks. In IFIP Wireless Days. (2010)

Kondareddy, Y. R., Agrawal, P. y Sivalingam, K.; Cognitive radio network setup without a common control channel. In IEEE Military Communications Conference MILCOM. (2008)

Lee, W. Y. y Akyildiz, I. F.; Optimal spectrum sensing framework for cognitive radio networks. IEEE Transactions on Wireless Communications, 7(10), 3845-3857 (2008)

Li, X. y Zekavat, S. A.; Traffic Pattern Prediction and Performance Investigation for Cognitive Radio Systems. In 2008 IEEE Wireless Communications and Networking Conference 894-899 (2008)

Lin, Y. B. y Ai-Chun Pang; Comparing soft and hard handoffs. IEEE Transactions on Vehicular Technology, 49(3), 192-798 (2000)

Liu, Y. y Tewfik, A.; Primary Traffic Characterization and Secondary Transmissions. IEEE Transactions on Wireless Communications, 13(6), 3003-3016 (2014)

López, D. A., García, N. Y. y Herrera, J. F.; Desarrollo de un Modelo Predictivo para la Estimación del Comportamiento de Variables en una Infraestructura de Red, doi: 10.4067/S0718-07642015000500018, Inf. tecnol. (en línea), 26(5), 143-154 (2015)

López, D. A., Trujillo, E. R. y Gualdron, O. E.; Elementos Fundamentales que Componen la Radio Cognitiva y Asignación de Bandas Espectrales, doi: 10.4067/S0718-07642015000100004, Inf. tecnol. (en línea), 26(1), 23-40 (2015)

Ma, L., Shen, C. C. y Ryu, B.; Single-radio adaptive channel algorithm for spectrum agile wireless ad hoc networks. In 2nd IEEE International Symposium on New Frontiers in Dynamic Spectrum Access Networks. 547-558 (2007)

Marinho, J. y Monteiro, E.; Cognitive radio: Survey on communication protocols, spectrum decision issues, and future research directions. Wireless Networks, 18(2), 147-164 (2012)

Masonta, M. T., Mzyece, M. y Ntlatlapa, N.; Spectrum Decision in Cognitive Radio Networks: A Survey. Communications Surveys Tutorials, IEEE, 15(3), 1088-1107 (2013)

NSF Workshop; Future Directions in Cognitive Radio Network Research. (2009)

Pedraza, L. F., Forero, F. y Paez, I. P. Evaluación de ocupación del espectro radioeléctrico en BogotáColombia. Ingenieria Y Ciencia, 10(19), 127-143 (2014)

Pla, V., Vidal, J. R., Martinez-Bauset, J. y Guijarro, L.; Modeling and characterization of spectrum white spaces for underlay cognitive radio networks. In IEEE International Conference on Communications. (2010)

Rahimian, N., Georghiades, C. N., Shakir, M. Z. y Qaraqe, K. A.; On the probabilistic model for primary and secondary user activity for OFDMA-based cognitive radio systems: Spectrum occupancy and system throughput perspectives. IEEE Transactions on Wireless Communications, 13(1), 356-369 (2014) 
Ramirez-Perez, C. y Ramos-R, V.; On the Effectiveness of Multi-criteria Decision Mechanisms for Vertical Handoff. In 27th International Conference on Advanced Information Networking and Applications (AINA) 1157-1164 (2013)

Rodriguez-Colina, E., Ramirez, P. C. y Carrillo, E.; Multiple attribute dynamic spectrum decision making for cognitive radio networks. In Eighth International Conference on Wireless and Optical Communications Networks. 1-5 (2011)

Sriram, K. y Whitt, W.; Characterizing Superposition Arrival Processes in Packet Multiplexers for Voice and Data. IEEE Journal on Selected Areas in Communications, 4(6), 833-846 (1986)

Stevens-Navarro, E., Martinez-Morales, J. D. y Pineda-Rico, U.; Evaluation of Vertical Handoff Decision Algorightms Based on MADM Methods for Heterogeneous Wireless Networks. Journal of Applied Research and Technology, 10(4), 534-548 (2012)

Stevens-Navarro, E. y Wong, V.; A vertical handoff decision algorithm for heterogeneous wireless networks. In IEEE Wireless Communications and Networking Conference 3199-3204 (2007)

Tragos, E. Z., Zeadally, S., Fragkiadakis, A. G. y Siris, V. a.; Spectrum assignment in cognitive radio networks: A comprehensive survey. IEEE Communications Surveys and Tutorials, 15(3), 1108-1135 (2013)

Van Quang, B., Prasad, R. V. y Niemegeers, I.; A survey on handoffs - Lessons for $60 \mathrm{GHz}$ based wireless systems. IEEE Communications Surveys and Tutorials, 14(1), 64-86 (2012)

Wang, C. W. y Wang, L. C.; Modeling and analysis for proactive-decision spectrum handoff in cognitive radio networks. In IEEE International Conference on Communications. 1-6 (2009)

Wang, C. W., Wang, L. C. y Adachi, F. Modeling and analysis for reactive-decision spectrum handoff in cognitive radio networks. In GLOBECOM - IEEE Global Telecommunications Conference. 1-6 (2010)

Wang, L. C. y Wang, C. W.; Spectrum handoff for cognitive radio networks: Reactive-sensing or proactivesensing? In IEEE International Conference Performance, Computing, and Communications. 343-348 (2008)

Wang, L. C., Wang, C. W. y Chang, C. J.; Modeling and Analysis for Spectrum Handoffs in Cognitive Radio Networks. IEEE Transactions on Mobile Computing, 11(9), 1499-1513 (2012)

Wang, X., Wong, A. y Ho, P. H.; Dynamically optimized spatiotemporal prioritization for spectrum sensing in cooperative cognitive radio. Wireless Networks, 16(4), 889-901 (2010)

Wei, Q., Farkas, K., Prehofer, C., Mendes, P. y Plattner, B.; Context-aware handover using active network technology. Computer Networks, 50(15), 2855-2872 (2006)

Weingart, T., Sicker, D. C. y Grunwald, D.; A statistical method for reconfiguration of cognitive radios. IEEE Wireless Communications, 14(4), 34-40 (2007)

Willkomm, D., Machiraju, S., Bolot, J. y Wolisz, A.; Primary users in cellular networks: a large-scale measurement study. In IEEE Symposium on New Frontiers in Dynamic Spectrum Access Networks, DySPAN 401-411 (2008)

Xu, Y., Anpalagan, A., Wu, Q., Shen, L., Gao, Z. y Wang, J.; Decision-Theoretic Distributed Channel Selection for Opportunistic Spectrum Access: Strategies, Challenges and Solutions. IEEE Communications Surveys \& Tutorials, 15(4), 1689-1713 (2013) 
